\title{
Intrinsic and extrinsic epigenetic age acceleration are associated with hypertensive target organ damage in older African Americans
}

Jennifer A. Smith ${ }^{1,2^{*}} \mathbb{D}$, Jeremy Raisky ${ }^{1 \dagger}$, Scott M. Ratliff', Jiaxuan Liu', Sharon L. R. Kardia ${ }^{1}$, Stephen T. Turner ${ }^{3}$, Thomas H. Mosley ${ }^{4}$ and Wei Zhao ${ }^{1}$

\begin{abstract}
Background: Epigenetic age acceleration, a measure of biological aging based on DNA methylation, is associated with cardiovascular mortality. However, little is known about its relationship with hypertensive target organ damage to the heart, kidneys, brain, and peripheral arteries.
\end{abstract}

Methods: We investigated associations between intrinsic (IEAA) or extrinsic (EEAA) epigenetic age acceleration, blood pressure, and six types of organ damage in a primarily hypertensive cohort of 1390 African Americans from the Genetic Epidemiology Network of Arteriopathy (GENOA) study. DNA methylation from peripheral blood leukocytes was collected at baseline (1996-2000), and measures of target organ damage were assessed in a follow-up visit (2000-2004). Linear regression with generalized estimating equations was used to test for associations between epigenetic age acceleration and target organ damage, as well as effect modification of epigenetic age by blood pressure or sex. Sequential Oligogenic Linkage Analysis Routines (SOLAR) was used to test for evidence of shared genetic and/or environmental effects between epigenetic age acceleration and organ damage pairs that were significantly associated.

Results: After adjustment for sex, chronological age, and time between methylation and organ damage measures, higher IEAA was associated with higher urine albumin to creatinine ratio (UACR, $p=0.004$ ), relative wall thickness (RWT, $p=0.022$ ), and left ventricular mass index (LVMI, $p=0.007$ ), and with lower ankle-brachial index $(A B I, p=0.014)$. EEAA was associated with higher LVMI $(p=0.005)$. Target organ damage associations for all but IEAA with LVMI remained significant after further adjustment for blood pressure and antihypertensive use $(p<0.05)$. Further adjustment for diabetes attenuated the IEAA associations with UACR and RWT, and adjustment for smoking attenuated the IEAA association with ABI. No effect modification by age or sex was observed.

Conclusions: Measures of epigenetic age acceleration may help to better characterize the functional mechanisms underlying organ damage from cellular aging and/or hypertension. These measures may act as subclinical biomarkers for damage to the kidney, heart, and peripheral vasculature; however more research is needed to determine whether these relationships remain independent of lifestyle factors and comorbidities.

(Continued on next page)

\footnotetext{
* Correspondence: smjenn@umich.edu

${ }^{\dagger}$ Jennifer A. Smith and Jeremy Raisky contributed equally to this work.

'Department of Epidemiology, School of Public Health, University of

Michigan, Ann Arbor, Ml 48109, USA

${ }^{2}$ Survey Research Center, Institute for Social Research, University of Michigan,

Ann Arbor, Ml 48104, USA

Full list of author information is available at the end of the article
}

(c) The Author(s). 2019 Open Access This article is distributed under the terms of the Creative Commons Attribution 4.0 International License (http://creativecommons.org/licenses/by/4.0/), which permits unrestricted use, distribution, and reproduction in any medium, provided you give appropriate credit to the original author(s) and the source, provide a link to the Creative Commons license, and indicate if changes were made. The Creative Commons Public Domain Dedication waiver (http://creativecommons.org/publicdomain/zero/1.0/) applies to the data made available in this article, unless otherwise stated. 
(Continued from previous page)

Keywords: Epigenetic age, Age acceleration, DNA methylation, Target organ damage, Hypertension, Estimated glomerular filtration rate, Urinary albumin-creatinine ratio, Left ventricular mass, Relative wall thickness, White matter hyperintensity, Ankle-brachial index

\section{Background}

Nearly one third of American adults have hypertension [1], and African Americans are affected disproportionately ( $43 \%$ of men and $46 \%$ of women) [2]. Over time, hypertension can lead to damage in target organs, including the heart, brain, kidneys, and peripheral vasculature [3]. Recently, genome-wide association studies have begun to identify genetic risk factors for the development of specific types of hypertensive target organ damage [4-7], and large-scale epigenome-wide studies for epigenetic risk factors are underway. However, additional biomarkers are needed to better characterize and predict the type, severity, and progression of target organ damage across individuals in this era of precision medicine. A deeper understanding of etiology and enhanced risk prediction may also help to alleviate hypertension-related health disparities.

Inter-individual differences in target organ damage may be a consequence of biological, or physiological, aging due to genetic and/or environmental factors such as inflammation, oxidative stress, or other cellular mechanisms [8]. As described by Karasik et al., biological age is a measure of the functional status of an individual relative to others with the same chronological age group [9]. Biological aging can be estimated using a variety of biomarkers such as functional performance metrics (e.g., measures of frailty), blood biochemistry (e.g., inflammatory markers), telomere length, and, more recently, epigenetics. Measures of biological aging, such as telomere shortening, have been shown to be associated with subclinical measures of function or morphology in the heart $[8,10]$, kidneys [11], and peripheral vasculature [10]. Epigenetic mechanisms, including DNA methylation, histone acetylation, and microRNA, play a key role in the regulation of gene expression, which is known to change with age [12]. Recently, several methods have been developed that use DNA methylation, cytosine- 5 methylation of $\mathrm{CPG}$ dinucelotides, to estimate epigenetic age acceleration, the difference between biological and chronological age [13, 14].

Epigenetic age acceleration is significantly associated with a variety of age-related conditions including allcause mortality [15], cancer incidence and mortality [16], cardiovascular mortality [17], Alzheimer's disease [18], Parkinson's Disease [19], Huntington's Disease [20], and menopause [21]. It is also associated with tissue aging [22] and cellular senescence [13, 23]. In this study, we evaluate two widely utilized measures of age acceleration, intrinsic epigenetic age acceleration (IEAA) and extrinsic epigenetic age acceleration (EEAA). IEAA captures cellular age acceleration independently of blood cell proportions, which are known to change with age. EEAA incorporates intrinsic measures as well as blood cell proportions. To date, only a few studies have investigated the association between epigenetic age acceleration and measures of target organ damage [24, 25]. It is now imperative to replicate these findings in independent populations, to examine additional measures, and to investigate whether the effects of age acceleration on target organ damage are modified by other factors.

Chronic inflammation and changes in the adaptive and innate immune response are key features of hypertension and consequent target organ damage. Peripheral blood leukocytes, which interact with peripheral tissues via excretion of cytokines and other cellular signals, are clearly implicated in target organ damage, although additional research is needed to precisely define the role of specific immune cell populations [26]. Since leukocytes provide a regulatory mechanism of inflammation due to hypertension across organ systems, peripheral blood leukocytes are an ideal cell type for exploring the relationship between hypertension and epigenetic biomarkers of age. In this study, we evaluated whether epigenetic age acceleration is associated with target organ damage to the heart, brain, kidneys, and peripheral vasculature of 1390 African Americans. For measures of target organ damage that were associated with epigenetic age acceleration, we also evaluated whether the effects were modified by systolic blood pressure (SBP), diastolic blood pressure (DBP), or sex. Finally, we used pedigree-based biometric methods to estimate heritability of the epigenetic age acceleration and target organ damage measures, and to assess whether associations between age acceleration and organ damage were due primarily to shared genetic or environmental influences.

\section{Methods \\ Study sample}

The Genetic Epidemiology Network of Arteriopathy (GENOA) study consists of hypertensive sibships that were recruited for linkage and association studies in order to identify genes that influence blood pressure and its target organ damage [27]. In the initial phase of GENOA (Phase I: 19962001), all members of sibships containing $\geq 2$ individuals with 
essential hypertension clinically diagnosed before age 60 were invited to participate, including both hypertensive and normotensive siblings. A total of 1583 non-Hispanic whites from Rochester, MN, and 1854 African Americans from Jackson, MS, were enrolled. In the second phase of GENOA (Phase II: 2000-2004), 1239 non-Hispanic white and 1482 African American participants were successfully re-recruited to measure potential target organ damage due to hypertension. DNA methylation was measured using blood samples collected at Phase I for 1416 African Americans. After removing outliers ( $>4 \mathrm{SD}$ from the mean) and those with missing covariates, 1390 participants from 618 sibships also had measures of target organ damage collected at Phase II and/ or in an ancillary study of brain MRI conducted shortly after Phase II.

\section{Methylation measures}

Genomic DNA from 1416 participants was extracted from peripheral blood leukocytes, bisulfite converted, and then measured for DNA methylation on Illumina Infinium HumanMethylation BeadChips ( $N=316$ from $450 \mathrm{~K}$ and $N=1100$ from EPIC) using stored blood samples collected at Phase I. IDAT files were imported using the Minfi R package [28], and the shinyMethyl $\mathrm{R}$ package [29] was used to exclude sex mismatches and outliers. Probes with detection $p$-value $<10^{-16}$ were considered to be successfully detected [30], and samples and probes with detection rate $<10 \%$ were removed. Samples with incomplete bisulfite conversion identified using the QCinfo function in the ENmix R package [31] were removed. The proportions of each white blood cell type within the blood sample were estimated using Houseman's method [32].

\section{Epigenetic age acceleration}

Methylation beta values were used to estimate the DNA methylation age prior to normalization, separately for the $450 \mathrm{~K}$ and EPIC data, using the Horvath epigenetic age calculator (https://dnamage.genetics.ucla.edu) [13]. This calculator estimates a cumulative epigenetic aging index that predicts DNA methylation age (DNAm age). The residual from regressing DNAm age on chronological age provides a measure of epigenetic age acceleration. Horvath, et al. (2013) [13] and Hannum et al. (2013) [14] have developed independent methods that use different sets of CpG sites to estimate DNAm age, herein referred to as Horvath DNAm age and Hannum DNAm age. Intrinsic epigenetic age acceleration (IEAA) is the residual from a multivariable regression of Horvath DNAm age, estimated using $353 \mathrm{CpGs}$ specified in Horvath, et al. (2013) [13], on chronological age and blood cell count estimates. This metric is independent of age-related changes in blood cell composition that are characteristic of immune system aging. It captures cell- intrinsic properties of aging with preservation across differing cell types and organs that likely indicates a fundamental aging process $[33,34]$.

To calculate extrinsic epigenetic age acceleration (EEAA), 71 CpGs specified in Hannum, et al. (2013) [14] are used to calculate Hannum DNAm age, which is then combined with three blood cell components (naïve cytotoxic $\mathrm{T}$ cells, exhausted cytotoxic $\mathrm{T}$ cells, and plasmablasts) to form an aggregate measure (enhanced Hannum DNAm age). EEAA is the residual from a regression of enhanced Hannum DNAm age onto chorological age. This measure captures both intrinsic epigenetic age as well as the weighted average of age-related characteristic changes in blood cell composition such as decreases in naive CD8+ T cells and increases in memory or exhausted CD8+ T cells [19]. Whereas IEAA is designed to be independent of blood cell counts, EEAA incorporates them, and is thus a measure of immune system aging.

We note that the EPIC array does not include all of the $\mathrm{CpG}$ sites that were originally used to construct the Horvath DNAm age (EPIC is missing 19 sites) and Hannum DNAm age (EPIC is missing 6 sites). Previous studies have demonstrated that this does not substantively compromise the performance of the DNAm age predictors [35]. Further, epigenetic age acceleration measurements such as EEAA and IEAA, which reflect the relative differences among the measured population, are especially robust to this difference in calculation.

Using the small number of samples $(n=102)$ that had methylation measured on both the $450 \mathrm{~K}$ and EPIC arrays, we confirmed that both epigenetic age and epigenetic age acceleration calculated from the two different array types showed relatively strong correlation $(r=0.88$ for Horvath DNAm age, $r=0.95$ for Hannum DNAm age, $r=0.70$ for IEAA, $r=0.84$ for EEAA, all $p<0.05$ ). Thus, we combined data from participants with $450 \mathrm{~K}$ and EPIC measures of epigenetic age acceleration into a single analysis sample. For those measured on both arrays, we used the age acceleration measures calculated from the EPIC array.

\section{Clinical assessments and covariate definitions}

Height was measured by stadiometer, and weight by electronic balance. Body mass index (BMI) was calculated as weight in kilograms divided by the square of height in meters. Resting systolic blood pressure (SBP) and diastolic blood pressure (DBP) were measured by a random zero sphygmomanometer and a cuff appropriate for arm size. The second and third of three readings, taken after the participant sat for at least $5 \mathrm{~min}$, were averaged for analysis. Participants were characterized as current, former, or never smokers. Hypertension was defined as $\mathrm{SBP} \geq 140$ $\mathrm{mmHg}, \quad \mathrm{DBP} \geq 90 \mathrm{mmHg}$, or self-reported physiciandiagnosed hypertension and current antihypertensive 
medication use. Diabetes was defined as fasting serum glucose concentration $\geq 126 \mathrm{mg} / \mathrm{dl}$ or self-reported physiciandiagnosed diabetes and current anti-diabetes medication use (insulin or hypoglycemic agents).

\section{Kidney function}

Blood was drawn after an overnight fast of at least $8 \mathrm{~h}$, and urine was collected on the morning of the study visit. Serum creatinine, urine creatine, and urine albumin were assessed with enzymatic assays on a Hitachi 911 Chemistry Analyzer (Roche Diagnostics, Indianapolis, IN). Estimated glomerular filtration rate (eGFR) was calculated using serum creatinine [36]. Microalbuminuria was defined as urinary albumin-to-creatinine ratio (UACR) $\geq 30 \mathrm{mg} / \mathrm{g}$ but $<300 \mathrm{mg} / \mathrm{g}$, and macroalbuminuria was defined as UACR $\geq 300 \mathrm{mg} / \mathrm{g}$ [37].

\section{Ankle-brachial index $(A B I)$}

ABI was measured from participants in the supine position following a 5-min rest [38]. Appropriately sized BP cuffs were placed on each arm and ankle, and a Doppler ultrasound instrument was used to detect pulse. The cuff was inflated to $10 \mathrm{mmHg}$ above SBP and deflated at 2 $\mathrm{mmHg} / \mathrm{s}$. The first reappearance of the pulse was taken as SBP. To calculate ABI, the SBP at each site (posterior tibial and dorsalis pedis) bilaterally was divided by the higher of the two brachial SBPs (one on each side of the body). The lowest of the four ratios was designated as the ABI.

\section{Echocardiography}

Relative wall thickness (RWT) and left ventricular mass index (LVMI) were estimated as previously described [39]. Briefly, doppler, two-dimensional (2D), and Mmode (2D-guided) echocardiograms were performed following a standardized protocol [40]. Measurements were made at the echocardiography reading center using a computerized review station equipped with a digitizing tablet and monitor overlay used for calibration and quantification (Digisonics, Inc., Houston, Texas). Left ventricular linear dimensions were measured by $\mathrm{M}$ mode or 2D echocardiography according to American Society of Echocardiography recommendations [41, 42]. Left ventricular mass (LVM) was calculated using enddiastolic dimensions by an anatomically validated formula, and RWT was calculated as twice the posterior wall thickness divided by the left ventricular internal dimension [43]. Left ventricular mass index was then calculated by dividing LVM by height in meters raised to the 2.7 th power.

\section{White matter hyperintensity}

White matter hyperintensity (WMH) was measured at a separate study visit approximately 1 year after the
Phase II examination. Brain magnetic resonance imaging (MRI) was performed using Signa $1.5 \mathrm{~T}$ MRI scanners (GE Medical Systems, Waukesha, WI, USA), and images were processed at Mayo Clinic [44]. Total brain and WMH volume in the corona-radiata and periventricular zone was determined from axial fluidattenuated inversion recovery (FLAIR) images [45]. Brain scans with cortical infarctions were excluded from the analyses because of the distortion of the WMH volume estimates that would be introduced in the automated segmentation algorithm. For additional details, see Smith et al. [46].

\section{Statistical analysis}

Target organ damage measures that were not normally distributed, UACR and WMH, were natural log transformed as $\ln (\mathrm{x})$ and $\ln (\mathrm{x}+1)$, respectively.

\section{Regression modeling}

Regression modeling was conducted using SAS software, Version 9.4 (Cary, NC). Generalized estimating equations (GEE) accounting for family structure were used to test the association between age acceleration (IEAA or EEAA) and blood pressure or target organ damage. Model 1 adjustment variables included age, sex, and the time between DNA methylation and target organ damage measurement. Model 2 also included SBP, DBP, and antihypertensive medication use. Model 3 further included BMI, diabetes, and smoking status. Analyses for echocardiographic measures also included microalbuminuria $(300 \mathrm{mg} / \mathrm{g}>\mathrm{UACR} \geq 30 \mathrm{mg} / \mathrm{g}$ ) and macroalbuminuria (UACR $\geq 300 \mathrm{mg} / \mathrm{g}$ ) in Model 3 [47]. Furthermore, all models with WMH also included total intracranial volume (TIV). Age and other adjustment variables were concurrent with target organ damage measurement. If the association between epigenetic age acceleration and target organ damage lost significance in Model 3, we also evaluated which of the adjustment variables (BMI, diabetes, or smoking) was responsible for this attenuation by testing the addition of each variable separately to Model 2. For target organ damage measures that were significantly associated with age acceleration $(p<0.05)$, we examined the interaction between age acceleration and sex, SBP, or DBP using the same covariates as described previously. Since we tested six related target organ damage traits with a prior hypothesis for each of them, we were interested in significant results at both a nominal $p$-value $(p<0.05)$ as well as a Bonferroni corrected p-value (0.0083). We note that the Bonferroni approach is conservative in this setting, since the measures of target organ damage as well as the measures of epigenetic age acceleration are not independent. 


\section{Sensitivity analyses}

Given that the full sample included individuals with epigenetic age acceleration estimated from either the EPIC or the $450 \mathrm{~K}$ array, we evaluated whether adding array type as a covariate and/or limiting our sample to only those individuals with EPIC data $(N=1074)$ substantively influenced our major findings. Next, since educational attainment or other markers of socioeconomic status may influence both the epigenome and the development of target organ damage, we evaluated the impact of adjusting for educational attainment ( $<$ high school, high school, college or above) in Models 1-3. Finally, since subclinical measures of damage to target organ systems may be due to hypertension as well as other age-related processes, we first conducted analyses in the full sample regardless of hypertension status. As a sensitivity analysis, we also evaluated whether the relationships between epigenetic age acceleration and target organ damage measures remained consistent in the subsample of participants with hypertension $(N=1127)$.

\section{Heritability and genetic correlation}

Capitalizing on the sibship structure of GENOA, Sequential Oligogenic Linkage Analysis Routines (SOLAR) v6.6.2 [48] was used to estimate the heritability of each measure of epigenetic age acceleration or target organ damage measure, adjusting for each set of covariates in Models 1-3 described above. For linear regression models that showed a significant $(p<0.05)$ association between a measure of target organ damage and epigenetic age acceleration, we investigated whether the association was due primarily to shared genetic or environmental influences. SOLAR was used to estimate the proportion of phenotypic correlation $(\rho \mathrm{P})$ due to shared genetic influences (genetic correlation, $\rho G$ ) or environmental influences (environmental correlation, $\rho E$ ) between the two traits, using the same covariates as regression Models 1-3.

\section{Results}

Descriptive characteristics of the study participants are shown in Table 1. The average age at Phase I was 58.0 $( \pm 10.1)$ years, and the majority of the sample was female (71.1\%). The average amount of time between methylation measurement (Phase I) and target organ damage measurement (Phase II) was 5.1 years, except for WMH (6.3 years). DNA methylation age was $55.0( \pm 9.9)$ years at Phase I. Additional file 1: Figure S1 shows scatterplots of epigenetic age and epigenetic age acceleration measures between the $450 \mathrm{~K}$ and EPIC arrays from $102 \mathrm{du}-$ plicated samples. Histograms of IEAA and EEAA are provided in Additional file 1: Figure S2.

Neither IEAA nor EEAA was associated with SBP or DBP in linear regression analysis, except that higher EEAA
Table 1 Descriptive characteristics of GENOA African Americans

\begin{tabular}{|c|c|c|}
\hline Characteristic & Non-Missing $N$ & Mean (SD) or \% \\
\hline Age at Phase I (years) & 1390 & $58.0(10.1)$ \\
\hline DNAm age at Phase I (years) & 1390 & $55.0(9.9)$ \\
\hline IEAA & 1389 & $0.1(4.8)$ \\
\hline EEAA & 1390 & $0.2(5.8)$ \\
\hline Time between Phases I and II & 1390 & $5.1(1.3)$ \\
\hline Sex & 1390 & \\
\hline Male & & $28.9 \%$ \\
\hline Female & & $71.1 \%$ \\
\hline Smoking & 1390 & \\
\hline Never & & $60.2 \%$ \\
\hline Former & & $27.6 \%$ \\
\hline Current & & $12.2 \%$ \\
\hline BMI $\left(\mathrm{kg} / \mathrm{m}^{2}\right)$ & 1390 & $31.7(6.7)$ \\
\hline Systolic BP (mmHg) & 1390 & $138.5(20.8)$ \\
\hline Diastolic BP (mmHg) & 1390 & $79.5(10.9)$ \\
\hline Antihypertensive use & 1390 & \\
\hline No & & $29.2 \%$ \\
\hline Yes & & $70.8 \%$ \\
\hline Diabetes & 1390 & \\
\hline No & & $69.7 \%$ \\
\hline Yes & & $30.3 \%$ \\
\hline Albuminuria (mg/g) & 1390 & \\
\hline Normal & & $81.7 \%$ \\
\hline Micro $^{a}$ & & $14.3 \%$ \\
\hline Macro $^{b}$ & & $4.0 \%$ \\
\hline eGFR $\left(\mathrm{mL} / \mathrm{min} / 1.73 \mathrm{~m}^{2}\right)$ & 1390 & $88.9(21.0)$ \\
\hline UACR (mg/g) & 1390 & $68.4(352.9)$ \\
\hline RWT & 1352 & $0.3(0.1)$ \\
\hline LVMI $\left(\mathrm{g} / \mathrm{m}^{2.7}\right)$ & 1346 & $39.3(10.4)$ \\
\hline$A B \mid$ & 1359 & $1.0(0.1)$ \\
\hline WMH $\left(\mathrm{cm}^{3}\right)^{c}$ & 758 & $10.6(11.6)$ \\
\hline $\operatorname{TIV}\left(\mathrm{cm}^{3}\right)^{\mathrm{c}}$ & 766 & 1373.9 (135.8) \\
\hline
\end{tabular}

Abbreviations: IEAA Intrinsic epigenetic age acceleration, EEAA Extrinsic epigenetic age acceleration, BMI Body mass index, BP Blood pressure, TIV Total intracranial volume, eGFR Estimated glomerular filtration rate, UACR Urinary albumin to creatinine ratio, LVMI Left ventricular mass index, RWT Relative wall thickness, ABI Ankle-brachial index, WMH White matter hyperintensity, TIV Total intracranial volume

${ }^{a}$ Microalbuminuria is defined as $30 \mathrm{mg} / \mathrm{g} \leq \mathrm{UACR}<300 \mathrm{mg} / \mathrm{g}$

${ }^{b}$ Macroalbuminuria is defined as UACR $\geq 300 \mathrm{mg} / \mathrm{g}$

'WMH and TIV were measured an average of 6.3 years after Phase I

All measures were taken at Phase II unless otherwise noted

was associated with increased SBP in Model $2(p=0.015$, Additional file 1: Table S1). Table 2 presents results from the association between IEAA or EEAA and each of the six target organ damage measures, separately. Higher IEAA was associated with higher UACR in Models $1(p=0.004)$ and $2(p=0.036)$; however, the association was attenuated 
Table 2 Regression results for the association between epigenetic age acceleration and target organ damage measures among GENOA African Americans

\begin{tabular}{|c|c|c|c|c|c|c|c|}
\hline \multirow{2}{*}{$\begin{array}{l}\text { Target organ } \\
\text { damage }\end{array}$} & \multirow{2}{*}{$\begin{array}{l}\text { Epigenetic } \\
\text { age } \\
\text { acceleration }\end{array}$} & \multicolumn{2}{|l|}{ Model 1} & \multicolumn{2}{|l|}{ Model 2} & \multicolumn{2}{|l|}{ Model 3} \\
\hline & & $\bar{\beta}$ & $P$ & $\bar{\beta}$ & $P$ & $\bar{\beta}$ & $P$ \\
\hline \multirow[t]{2}{*}{ eGFR $(n=1389)$} & IEAA & -0.082 & 0.444 & -0.042 & 0.694 & -0.035 & 0.745 \\
\hline & EEAA & 0.119 & 0.180 & 0.095 & 0.284 & 0.089 & 0.320 \\
\hline \multirow[t]{2}{*}{$\operatorname{UACR}^{\mathrm{a}}(\mathrm{n}=1390)$} & IEAA & 0.023 & 0.004 & 0.017 & 0.036 & $8.9 E-3$ & 0.246 \\
\hline & EEAA & 0.010 & 0.187 & $7.2 \mathrm{E}-3$ & 0.287 & $1.5 \mathrm{E}-3$ & 0.826 \\
\hline \multirow[t]{2}{*}{ RWT $(n=1352)$} & IEAA & $6.2 \mathrm{E}-4$ & 0.022 & $5.4 \mathrm{E}-4$ & 0.041 & $4.5 E-4$ & 0.089 \\
\hline & EEAA & $-3.4 E-5$ & 0.886 & $-4.8 \mathrm{E}-5$ & 0.836 & $-1.2 \mathrm{E}-4$ & 0.598 \\
\hline \multirow[t]{2}{*}{ LVMI $(n=1346)$} & IEAA & 0.163 & 0.007 & 0.110 & 0.054 & 0.062 & 0.260 \\
\hline & EEAA & 0.131 & 0.005 & 0.119 & 0.009 & 0.093 & 0.029 \\
\hline \multirow[t]{2}{*}{$\mathrm{ABI}(n=1359)$} & IEAA & $-2.1 E-3$ & 0.014 & $-1.9 \mathrm{E}-3$ & 0.028 & $-1.6 \mathrm{E}-3$ & 0.057 \\
\hline & EEAA & $-1.2 \mathrm{E}-3$ & 0.075 & $-1.0 \mathrm{E}-3$ & 0.117 & $-8.4 \mathrm{E}-4$ & 0.196 \\
\hline \multirow[t]{2}{*}{$\mathrm{WMH}^{\mathrm{a}}(n=758)$} & IEAA & $-4.2 e-3$ & 0.333 & $-5.4 \mathrm{e}-3$ & 0.213 & $-7.8 e-3$ & 0.075 \\
\hline & EEAA & $6.8 e-3$ & 0.068 & $7.0 e-3$ & 0.053 & $5.7 e-3$ & 0.109 \\
\hline
\end{tabular}

Abbreviations: IEAA Intrinsic epigenetic age acceleration, EEAA Extrinsic epigenetic age acceleration, eGFR Estimated glomerular filtration rate, UACR Urinary albumin to creatinine ratio, RWT Relative wall thickness, LVMI Left ventricular mass index, $A B I$ Ankle-brachial index, WMH White matter hyperintensity Model 1: Target organ damage $=$ epigenetic age acceleration + chronological age + sex + time between methylation and target organ damage measure Model 2: Target organ damage $=$ Model 1 covariates + SBP + DBP + antihypertensive medication

Model 3: Target organ damage $=$ Model 2 covariates + smoking + diabetes $+\mathrm{BMI}$

Model 3 for RWT and LVM also includes microalbuminuria and macroalbuminuria

All models for WMH also include total intracranial volume (TIV)

${ }^{a}$ Variables were natural log transformed prior to analysis

Bold values correspond to beta values and $p$-values, where $p<0.05$

after additional adjustment for smoking, diabetes, and BMI (Model 3, $p=0.246$ ). In Model 1, a 1-year increase in IEAA was associated with 0.023 units increase in $\log \mathrm{UACR}$, corresponding to a $0.70 \mathrm{mg} / \mathrm{g}$ increase for a person with UACR of $30 \mathrm{mg} / \mathrm{g}$ (the threshold for microalbuminuria) and a $6.98 \mathrm{mg} / \mathrm{g}$ increase for a person with UACR of $300 \mathrm{mg} / \mathrm{g}$ (the threshold for macroalbuminuria). IEAA was not associated with eGFR.

Higher IEAA was significantly associated with higher RWT in Models 1 and 2 ( $p=0.022$ and 0.041 , respectively) and LVMI in Model $1(p=0.007)$. The association was only marginally significant in Model 3 for RWT $(p=0.089)$ and in Model 2 for LVMI $(p=0.054)$, and was fully attenuated in Model 3 for LVMI $(p=0.260)$. In Model 1, a 1-year increase in IEAA was associated with a 0.0006 unit increase in RWT and a $0.163 \mathrm{~g} / \mathrm{m}^{2.7}$ increase in LVMI. Higher IEAA was also associated with lower ABI in Models 1 and 2 ( $p=0.014$ and 0.028, respectively), but was only marginally significant in Model $3(p=0.057)$. In Model 1, a 1-year increase in IEAA was associated with 0.002 units decrease in ABI. IEAA was not associated with WMH. EEAA was associated with LVMI in Models $1(p=0.005), 2(p=0.009)$, and $3(p=$ 0.029 ), but not with other measures of organ damage. A 1 -year increase in EEAA was associated with a $0.163 \mathrm{~g} /$ $\mathrm{m}^{2.7}$ increase in LVMI. Among the five associations we observed for Model 1, three (UACR and IEAA, LVMI and IEAA, LVMI and EEAA) remained significant after Bonferroni correction for multiple testing. For organ damage measures that were significantly associated with age acceleration $(p<0.05)$, we examined the interaction between age acceleration and sex, SBP, or DBP; however, none of the interactions were significant (all $p>0.05$ ).

To investigate which variables were leading to the attenuation of the IEAA association with UACR, RWT, and ABI in Model 3, we examined the change in the beta coefficient for IEAA when adding each of the Model 3 adjustment variables separately to Model 2 (Additional file 1: Table S2). For UACR and RWT, adding diabetes to the model resulted in the largest percentage change of the IEAA beta coefficient (45.5 and $12.4 \%$, respectively), resulting in attenuation of the association $(p<0.05)$. For ABI, smoking changed the beta coefficient the most (15.9\%) and resulted in attenuation $(p<0.05)$.

As a sensitivity analysis, we evaluated the effect of adding array type as a covariate or excluding individuals whose epigenetic age estimates were calculated from the $450 \mathrm{~K}$ array (Additional file 1: Table S3). Adding array type as a covariate did not have any impact on effect estimates or $p$-values for any of the associations in Model 1. When we conducted analysis with the smaller sample measured on the EPIC array only $(N=1074)$, there are no substantive changes except that some previously significant signals are slightly attenuated 
(UACR and IEAA, $p=0.059$; LVMI and IEAA, $p=0.062$, $\mathrm{ABI}$ and IEAA, $p=0.107$ ), which may be due to the smaller sample size. Also, the association between WMH and EEAA attained significance $(p=0.04)$ when it had previously been marginally significant $(p=0.068)$. Therefore, we conclude that combining two array types did not substantively influence the results. Adding educational attainment as a covariate also did not substantively impact the findings, except for the slight attenuation of the relationship between $\mathrm{ABI}$ and IEAA in Model 2 ( $p=0.056$, Additional file 1: Table S4).

We also evaluated the same linear regression models in the subset of GENOA participants with hypertension ( $n=1127$; Additional file 1: Table S5). Regression results were substantively consistent for the relationship between IEAA and UACR, LVMI, and ABI. EEAA remained associated with LVMI in Models 1 and $2(p=$ 0.017 and $p=0.035)$, but the association was only marginally significant in Model $3(p=0.083)$. In contrast to the full sample, no relationship was observed between IEAA and RWT for any of the models in the subsample with hypertension. However, the association between EEAA and $\mathrm{WMH}$ was significant in the hypertensive sample in Models 1 and $2(p=0.040$ and $p=0.041$, respectively) and marginal in Model $3(p=0.071)$, whereas the associations had been only marginal in the full sample ( $p=0.068,0.053$, and 0.109 for Models 1, 2, and 3).

All of the measures of epigenetic age acceleration and target organ damage evaluated had significant heritability ( $\mathrm{h}^{2}$, all $p<0.01$, Additional file 1: Table S6). In Model $1, \mathrm{~h}^{2}$ for IEAA was 0.480 and for EEAA was 0.607 , and these estimates did not change substantially when additional covariates were added in Models 2 and 3. Of the target organ damage traits, LVMI had the highest $h^{2}$ (0.577) and RWT had the lowest (0.276) in Model 1, and these heritabilities also remained relatively stable when additional covariates were added in Models 2 and 3. For the pairs of epigenetic age acceleration and target organ damage traits that were significantly associated in linear regression $(p<0.05)$, we also evaluated whether there was significant evidence of genetic or environmental correlation. No estimates of genetic correlation were significant (all $\rho \mathrm{G}<0.1$ and $p>0.1$ ), and only two of the pairs had at least marginally significant evidence of environmental correlation $(\rho E=0.176$ for IEAA and UACR in Model $1, p<0.05 ; \rho \mathrm{E}=0.229$ for EEAA and LVMI in Model 1, $0.05<p<0.01$; Table 3).

\section{Discussion}

Epigenetic age acceleration has shown robust associations with a variety of biological aging traits, but has not been extensively evaluated for association with organ damage associated with aging and hypertension. This study explored the association between intrinsic (IEAA) and extrinsic (EEAA) measures of age acceleration measured by DNA methylation and target organ damage in African Americans, a population disproportionately affected by hypertension. We found that IEAA was associated with measures of damage in the kidney (UACR), heart (RWT), and peripheral arterial disease (ABI), even after accounting for blood pressure and antihypertensive use. In addition, EEAA was associated with damage to the heart (LVM), and was also associated with white matter damage in the brain (WMH) for those with clinical hypertension. However, the associations between

Table 3 Phenotypic, genetic, and environmental correlations between epigenetic age acceleration and target organ damage measures among GENOA African Americans

\begin{tabular}{|c|c|c|c|c|c|c|c|}
\hline Target organ damage & Epigenetic age accleration & Model & $h^{2}$ TOD & $h^{2}$ EAA & $\rho_{p}$ & $\rho_{G}$ & $\rho_{E}$ \\
\hline \multirow[t]{2}{*}{$\overline{U^{\prime} A C R^{a}}$} & IEAA & 1 & $0.383^{* * *}$ & $0.484^{* * *}$ & $0.067^{*}$ & -0.075 & $0.176^{*}$ \\
\hline & & 2 & $0.376^{* * *}$ & $0.510^{* * *}$ & $0.056^{*}$ & 0.066 & 0.050 \\
\hline \multirow[t]{2}{*}{ RWT } & IEAA & 1 & $0.265^{* * *}$ & $0.483^{* * *}$ & $0.060^{*}$ & 0.001 & 0.097 \\
\hline & & 2 & $0.246^{* * *}$ & $0.510^{* * *}$ & $0.056^{*}$ & 0.086 & 0.042 \\
\hline \multirow[t]{4}{*}{ LVMI } & IEAA & 1 & $0.563^{* * *}$ & $0.480^{* * *}$ & $0.064^{*}$ & -0.032 & 0.168 \\
\hline & EEAA & 1 & $0.566^{* * *}$ & $0.604^{* * *}$ & $0.062^{*}$ & -0.056 & $0.229^{\dagger}$ \\
\hline & & 2 & $0.506^{* * *}$ & $0.601^{* * *}$ & $0.066^{*}$ & -0.005 & 0.155 \\
\hline & & 3 & $0.440^{* * *}$ & $0.604^{* * *}$ & $0.056^{*}$ & -0.022 & 0.144 \\
\hline \multirow[t]{2}{*}{$A B \mid$} & IEAA & 1 & $0.354^{* * *}$ & $0.483^{* * *}$ & $-0.074^{* *}$ & -0.004 & -0.125 \\
\hline & & 2 & $0.355^{* * *}$ & $0.510^{* * *}$ & $-0.067^{*}$ & -0.037 & -0.092 \\
\hline
\end{tabular}

Abbreviations: IEAA Intrinsic epigenetic age acceleration, EEAA Extrinsic epigenetic age acceleration, UACR Urinary albumin to creatinine ratio, $R W T$ Relative wall thickness, LVMI Left ventricular mass index, $A B I$ Ankle-brachial index, $h^{2}$ Heritability estimate, $\rho_{P}$ Phenotypic correlation, $\rho_{G}$ Genetic correlation, $\rho_{E}=$ environmental correlation

Model 1 covariates: chronological age + sex + time between methylation and target organ damage measure

Model 2 covariates: Model 1 covariates $+\mathrm{SBP}+\mathrm{DBP}+$ antihypertensive medication

Model 3 covariates: Model 2 covariates + smoking + diabetes + BMI + albuminuria

aariables were natural log transformed prior to analysis

${ }^{\dagger} p<0.1,{ }^{*} p<0.05,{ }^{* *} p<0.01,{ }^{* * *} p<0.001$ 
RWT and IEAA as well as ABI and IEAA were not significant after Bonferroni correction for multiple testing (Model 1); thus these results should be interpreted with caution. Neither IEAA nor EEAA was associated with eGFR, the most commonly-used biomarker of kidney function. Further, the majority of our findings were attenuated after adjusting for BMI, diabetes, and smoking, indicating that the associations between epigenetic age and target organ damage should be considered suggestive. This indicates that age acceleration may have physiological consequences, or be acting as an important subclinical biomarker for physiological processes, that influence organs vulnerable to the consequences of hypertension. However, further research is needed to determine whether these relationships remain independent of lifestyle factors and comorbidities.

For the significant associations between age acceleration and target organ damage, the effect directions were as expected. Higher IEAA, indicating a more advanced cellular aging process than expected based on chronological age, was associated with more damage to the kidney, heart, and the peripheral arteries. Adjusting for blood pressure and anti-hypertensive medications did not attenuate the significance of these associations, except for with LVMI, indicating that the relationship between IEAA and the organ damage measures was not mediated by differences in blood pressure. In fact, neither IEAA nor EEAA was strongly associated with either SBP or DBP, and relationships between IEAA and most organ damage measures remained consistent in analyses that included only those with hypertension. Thus, interindividual differences in vulnerability to target organ damage as a result of aging and hypertension may be a consequence of cellular physiological aging processes that are relatively independent of hypertension severity as well as immune system aging (as measured by EEAA). On these points, our study is consistent with prior literature in two ways. First, that there has been little to no association observed between hypertension or blood pressure and IEAA or EEAA after controlling for BMI and lifestyle factors $[17,49]$. Second, like our study, previous studies have demonstrated relationships between hypertension-related endpoints such as WMH [24] and cardiovascular mortality [50] after controlling for blood pressure level.

For target organ damage traits in the kidney (UACR) and heart (RWT), the relationship with IEAA was attenuated after adjusting for diabetes, although it remained marginally significant $(p<0.1)$ for RWT. The two primary etiologies for chronic kidney disease (CKD) are diabetic nephropathy and hypertension [51], and kidney damage due to diabetic nephropathy is often first detected clinically through increased albuminuria (higher UACR) prior to declines in eGFR [52]. Likewise, diabetes is also an independent risk factor for higher LVMI and RWT in African Americans [53], so it is possible that the relationship between these traits and IEAA was due entirely to the relationship between diabetes and IEAA. Some previous studies have found that epigenetic age acceleration was not associated with glucose and/or diabetes status after adjusting for BMI and lifestyle risk factors, and was only weakly associated with other diabetes risk factors such as triglycerides [17, 49]. A recent longitudinal study, however, observed the association between epigenetic age acceleration and fasting glucose [54]. More studies are needed to elucidate the complicated interplay among epigenetic age acceleration, diabetes, and related target organ damage to the kidney and heart.

For ABI, a 1-year change in age acceleration (IEAA) was associated with 0.002 unit decrease. Peripheral arterial disease is defined as having an ABI outside the range of 1.0-1.4 [55], with values below 1.0 indicating narrowing of arteries in certain areas of the body due to calcification [56]. The relationship between IEAA and $A B I$ was attenuated after adjusting for smoking, which is a very strong risk factor for ABI. Even though self-reported smoking was not found to be associated with epigenetic age acceleration in previous literature [49, 57-59], many smoking-associated CpG sites were associated with methylation age acceleration [59, 60]. It has been hypothesized that biological indicators of smoking may represent susceptibility to more generalized environmental factors, including alcohol consumption and lifestyle factors, which may be partially responsible for the apparent mediating effect of smoking on the relationship between IEAA and ABI observed in this study. For both UACR and ABI, age acceleration increased variation explained by only approximately $1-2 \%$ in all models evaluated.

Few studies have evaluated the relationship between epigenetic age acceleration and target organ damage. However, two studies did find significant relationships between universal epigenetic age acceleration estimated using Horvath DNAm age acceleration and white matter abnormalities [24, 25]. In 713 African Americans aged 51 to 73, increased epigenetic age acceleration was associated with increased WMH burden, as measured by MRI, with an age acceleration increase of 1 year associated with a $\sim 1$ grade ( 0.007 on the log scale) increase of WMH [24]. In a study of 376 Mexican Americans aged 23-93 years, increased age acceleration was associated with reduced white matter integrity measured with diffusion tensor imaging [25]. These studies used measures of universal age acceleration, not IEAA and EEAA as was used in our study. However, we conducted a posthoc analysis and found that universal age acceleration measures were not associated with WMH in our study (data not shown). The lack of association in our study 
may be due to reduced variability of the brain measures or the higher prevalence of hypertension, diabetes, or other comorbidities in our study population. However, we did observe an association between EEAA and WMH in our analysis of hypertensives only.

Although the directions of effect for IEAA and EEAA were relatively consistent in this study, they had nonoverlapping significant associations with target organ damage measures. IEAA is independent of the immune blood cell counts, primarily captures cell-intrinsic methylation changes, and has been shown to be relatively independent of lifestyle factors [34]. EEAA, however, captures changes in both blood cell counts as well as cell-intrinsic methylation changes and has been shown to be more strongly correlated with lifestyle factors [34, 49]. A significant association between EEAA and LVMI may reflect the greater importance of white blood cell distributional changes that influence this trait as compared to other target organ damage measures. White blood cell distributions may be reflective of a variety of influences, including cumulative lifetime exposure to infection, lifestyle choices (diet, smoking, exercise), and social stressors (socioeconomic status, discrimination, chronic burden, neighborhood factors). In this study, we were only able to adjust for some of the potentially relevant lifestyle, social, and metabolic processes that may be associated with increased epigenetic aging.

The associations within the GENOA African American cohort are reflective of a population with a high burden of metabolic diseases, including hypertension, diabetes, and obesity. We also note that there was loss to follow-up between Phases 1 and 2 which was differential by cardiovascular risk. We found that those lost to follow-up were more likely to be older (average $=1.2$ years), male, and a current smoker (all $p<0.05$ ). They also had higher BMI (average $=0.58 \mathrm{~kg} / \mathrm{m}^{2}$ ) and lower eGFR (average $=6 \mathrm{~mL} /$ $\min / 1.73 \mathrm{~m}^{2}$ ), and were more likely to have hypertension and diabetes (all $p<0.05)$. All of this indicates that loss to follow-up was greater in those with higher risk of cardiovascular disease, and thus our study sample may not include those with the highest risk of developing target organ damage in this population. Finally, over $2 / 3$ of our sample consisted of women, so our results may be more relevant to this population; this is important to recognize since epigenetic aging rates in blood have been shown to be higher for men than women [17].

Previous studies have found that EEAA rates are lower for African Americans than non-Hispanic whites, but that IEAA rates are similar [17]. This is contrary to what would be expected given that African Americans experience higher rates of many age-related chronic diseases. Recently, a new DNAm age predictor has been developed that better captures the racial differences in aging that would be expected based on the disparities in health outcomes across race/ethnic groups (that is, African Americans have higher rates of epigenetic aging) [61, 62]. Future studies examining this new DNAm age predictor are warranted.

The heritability estimates of epigenetic age acceleration vary by age group, ranging from 0.39 to 0.74 in adults [13, 57, 63]. The estimated heritabilities in this study were similar (0.48 to 0.60$)$. Although measures of epigenetic age and target organ damage had high heritability, there was very little evidence for shared genetic influences for pairs of associated epigenetic and organ damage measures. The inability to detect shared effects may be due to the relatively low correlations across pairs (all significant phenotypic correlations were less than 0.08). This may reflect the inability to adjust for all of the potentially relevant lifestyle or metabolic factors, or a true lack of pleiotropy across epigenetic age and target organ damage traits. In addition, due to the crosssectional nature of this study, it is impossible to determine whether changes in epigenetic aging are causative or a consequence of hypertension and its subsequent target organ damage. However, there was an approximately five-year lag between the time that methylation data was collected (Phase I) and target organ damage measures (Phase II), which lends credibility to the casual nature of epigenetic aging on subsequent target organ damage. Yet we were not able to account for the duration of hypertension, which preceded epigenetic measures for over two thirds of the study population. Longitudinal studies with measures of epigenetic age at multiple timepoints may help to disentangle these temporal relationships.

An important limitation in this study was that blood pressure and target organ damage traits were measured at only one study visit. Since blood pressure is known to be highly variable and elevated during clinical visits, a better approach for accurate blood pressure measures may be to 24-h ambulatory blood pressure measures. These are available for only a subset of GENOA participants, however, so the sample size would have been prohibitory. Some of the measures of target organ damage, such as urinary albumin, are also highly variable and would be more accurate if measured on multiple days; however, UACR is a more stable biomarker than urinary albumin alone [64]. Finally, we note that antihypertensive medications have differential effects in adequately preventing or controlling target organ damage. For example, renin-angiotensin-aldosterone system-blocking agents (RAAS inhibitors) may be particularly effective in reducing damage to the kidneys and heart [64], so future studies may benefit from systematically evaluating the relationship between epigenetic age acceleration and target organ damage taking differences in antihypertensive drug classes into account. 


\section{Conclusions}

In conclusion, we have found suggestive evidence of an association with epigenetic age acceleration and target organ damage to the kidneys, heart, and peripheral arteries. Specifically, intrinsic measures of epigenetic aging were associated with urinary albumin-creatinine ratio, relative wall thickness in the heart, and ankle-brachial index. Extrinsic measures of epigenetic aging were associated with left ventricular mass index. However, most of these associations were attenuated after adjusting for blood pressure levels, BMI, diabetes, and smoking. Further research is needed to determine whether epigenetic age acceleration may have potential clinical utility in helping to quantify risk of target organ damage across organ systems.

\section{Supplementary information}

Supplementary information accompanies this paper at https://doi.org/10. 1186/s12920-019-0585-5.

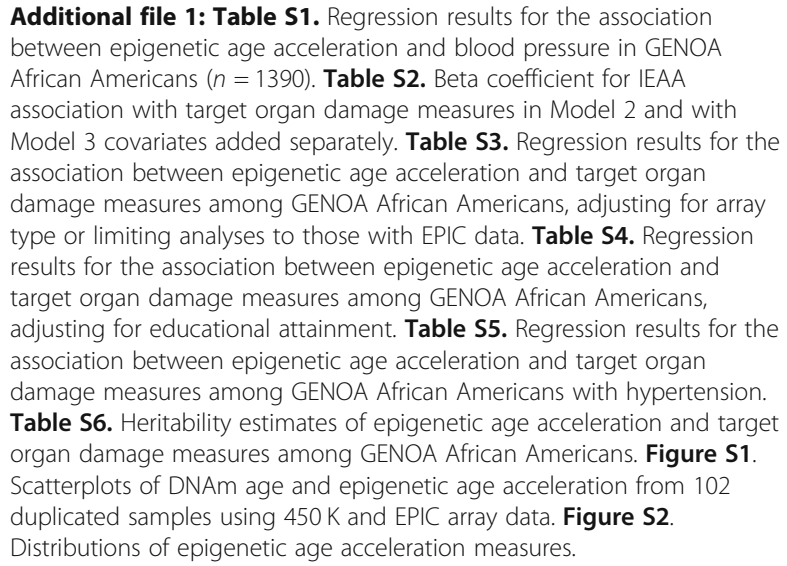
between epigenetic age acceleration and blood pressure in GENOA African Americans $(n=1390)$. Table S2. Beta coefficient for IEAA association with target organ damage measures in Model 2 and with Model 3 covariates added separately. Table S3. Regression results for the association between epigenetic age acceleration and target organ damage measures among GENOA African Americans, adjusting for array type or limiting analyses to those with EPIC data. Table S4. Regression results for the association between epigenetic age acceleration and target organ damage measures among GENOA African Americans, adjusting for educational attainment. Table S5. Regression results for the association between epigenetic age acceleration and target organ damage measures among GENOA African Americans with hypertension. Table S6. Heritability estimates of epigenetic age acceleration and target organ damage measures among GENOA African Americans. Figure S1. Scatterplots of DNAm age and epigenetic age acceleration from 102 duplicated samples using $450 \mathrm{~K}$ and EPIC array data. Figure S2. Distributions of epigenetic age acceleration measures.

\section{Abbreviations}

ABI: Ankle-brachial index; BMI: Body mass index; DBP: Diastolic blood pressure; DNAm: DNA methylation; EEAA: Extrinsic epigenetic age acceleration; eGFR: Estimated glomerular filtration rate; GENOA: Genetic Epidemiology Network of Arteriopathy; IEAA: Intrinsic epigenetic age acceleration; LVMI: Left ventricular mass index; RWT: Relative wall thickness; SBP: Systolic blood pressure; SOLAR: Sequential Oligogenic Linkage Analysis Routines; UACR: Urinary albumin-creatinine ratio; WMH: White matter hyperintensity

\section{Acknowledgements}

The authors wish to thank the staff and participants of the GENOA study.

\section{Authors' contributions}

JAS and JR jointly conceived and designed the study, and drafted the manuscript. WZ and JL cleaned the methylation data. JR and SMR conducted analyses, with assistance from WZ. STT measured the DNA methylation, THM led the collection of target organ damage phenotypes, and SLRK oversaw data collection at all phases of GENOA and assisted with manuscript revisions. JAS supervised the study and provided funding for analysis. All authors read and approved the final manuscript.

\section{Funding}

Support for the Genetic Epidemiology Network of Arteriopathy (GENOA) was provided by the National Heart, Lung and Blood Institute (HL054457, HL100185, HL119443, and HL133221) and the National Institute of
Neurological Disorders and Stroke (NS041558) of the National Institutes of Health. Funding agencies played no role in study design, data collection, analysis, interpretation of results, or writing the manuscript.

\section{Availability of data and materials}

The datasets used and/or analyzed in the current study are available from the corresponding author on reasonable request.

\section{Ethics approval and consent to participate}

Written informed consent was obtained from all participants, and Institutional Review Boards at the University of Michigan, University of Mississippi Medical Center, and Mayo Clinic approved this study.

\section{Consent for publication}

Not applicable.

\section{Competing interests}

The authors declare that they have no competing interests.

\section{Author details}

${ }^{1}$ Department of Epidemiology, School of Public Health, University of Michigan, Ann Arbor, MI 48109, USA. ²Survey Research Center, Institute for Social Research, University of Michigan, Ann Arbor, Ml 48104, USA. ${ }^{3}$ Division of Nephrology and Hypertension, Mayo Clinic, Rochester, MN 55905, USA.

${ }^{4}$ Memory Impairment and Neurodegenerative Dementia (MIND) Center,

University of Mississippi Medical Center, Jackson, MS 39126, USA.

Received: 8 October 2018 Accepted: 11 September 2019

Published online: 22 October 2019

\section{References}

1. Merai R, Siegel C, Rakotz M, Basch P, Wright J, Wong B, DHSC, Thorpe P. CDC grand rounds: a public health approach to detect and control hypertension. MMWR Morb Mortal Wkly Rep. 2016;65(45):1261-4.

2. Mozaffarian D, Benjamin EJ, Go AS, Arnett DK, Blaha MJ, Cushman M, de Ferranti S, Despres JP, Fullerton HJ, Howard VJ, et al. Heart disease and stroke statistics--2015 update: a report from the American Heart Association. Circulation. 2015;131(4):e29-322.

3. Nadar SK, Tayebjee MH, Messerli F, Lip GY. Target organ damage in hypertension: pathophysiology and implications for drug therapy. Curr Pharm Des. 2006:12(13):1581-92.

4. Murabito JM, White CC, Kavousi M, Sun W, Feitosa MF, Nambi V, Lamina C, Schillert A, Coassin S, Bis JC, et al. Association between chromosome 9p21 variants and the ankle-brachial index identified by a meta-analysis of 21 genome-wide association studies. Circ Cardiovasc Genet. 2012;5(1):100-12.

5. Pattaro C, Kottgen A, Teumer A, Garnaas M, Boger CA, Fuchsberger C Olden $M$, Chen MH, Tin A, Taliun D, et al. Genome-wide association and functional follow-up reveals new loci for kidney function. PLoS Genet. 2012;8(3):e1002584.

6. Verhaaren BF, Debette S, Bis JC, Smith JA, Ikram MK, Adams HH, Beecham AH, Rajan KB, Lopez LM, Barral S, et al. Multiethnic genome-wide association study of cerebral white matter hyperintensities on MRI. Circ Cardiovasc Genet. 2015:8(2):398-409.

7. Fox ER, Musani SK, Barbalic M, Lin H, Yu B, Ogunyankin KO, Smith NL, Kutlar A, Glazer NL, Post WS, et al. Genome-wide association study of cardiac structure and systolic function in African Americans: the candidate Gene Association resource (CARe) study. Circ Cardiovasc Genet. 2013;6(1):37-46.

8. Fuster JJ, Diez J, Andres V. Telomere dysfunction in hypertension. J Hypertens. 2007:25(11):2185-92.

9. Karasik D, Demissie S, Cupples LA, Kiel DP. Disentangling the genetic determinants of human aging: biological age as an alternative to the use of survival measures. J Gerontol A Biol Sci Med Sci. 2005;60(5):574-87.

10. Mwasongwe S, Gao Y, Griswold M, Wilson JG, Aviv A, Reiner AP, Raffield LM. Leukocyte telomere length and cardiovascular disease in African Americans: the Jackson heart study. Atherosclerosis. 2017;266:41-7.

11. Bansal N, Whooley MA, Regan M, McCulloch CE, Ix JH, Epel E, Blackburn E, Lin J, Hsu CY. Association between kidney function and telomere length: the heart and soul study. Am J Nephrol. 2012;36(5):405-11.

12. Peters MJ, Joehanes R, Pilling LC, Schurmann C, Conneely KN, Powell J, Reinmaa E, Sutphin GL, Zhernakova A, Schramm K, et al. The transcriptional landscape of age in human peripheral blood. Nat Commun. 2015;6:8570. 
13. Horvath S. DNA methylation age of human tissues and cell types. Genome Biol. 2013;14(10):R115.

14. Hannum G, Guinney J, Zhao L, Zhang L, Hughes G, Sadda S, Klotzle B, Bibikova M, Fan JB, Gao Y, et al. Genome-wide methylation profiles reveal quantitative views of human aging rates. Mol Cell. 2013;49(2):359-67.

15. Marioni RE, Shah S, McRae AF, Chen BH, Colicino E, Harris SE, Gibson J, Henders AK, Redmond P, Cox SR, et al. DNA methylation age of blood predicts all-cause mortality in later life. Genome Biol. 2015;16:25,015-0584-6.

16. Zheng Y, Joyce BT, Colicino E, Liu L, Zhang W, Dai Q, Shrubsole MJ, Kibbe WA, Gao T, Zhang Z, et al. Blood epigenetic age may predict Cancer incidence and mortality. EBioMedicine. 2016;5:68-73.

17. Horvath S, Gurven M, Levine ME, Trumble BC, Kaplan H, Allayee H, Ritz BR, Chen B, Lu AT, Rickabaugh TM, et al. An epigenetic clock analysis of race/ethnicity, sex, and coronary heart disease. Genome Biol. 2016;17(1):171,016-1030-0.

18. Levine ME, Lu AT, Bennett DA, Horvath S. Epigenetic age of the pre-frontal cortex is associated with neuritic plaques, amyloid load, and Alzheimer's disease related cognitive functioning. Aging (Albany NY). 2015;7(12):1198-211.

19. Horvath S, Ritz BR. Increased epigenetic age and granulocyte counts in the blood of Parkinson's disease patients. Aging (Albany NY). 2015;7(12):1130-42.

20. Horvath S, Langfelder P, Kwak S, Aaronson J, Rosinski J, Vogt TF, Eszes M, Faull RL, Curtis MA, Waldvogel HJ, et al. Huntington's disease accelerates epigenetic aging of human brain and disrupts DNA methylation levels. Aging (Albany NY). 2016;8(7):1485-512.

21. Levine ME, Lu AT, Chen BH, Hernandez DG, Singleton AB, Ferrucci $L$, Bandinelli S, Salfati E, Manson JE, Quach A, et al. Menopause accelerates biological aging. Proc Natl Acad Sci U S A. 2016;113(33):9327-32.

22. Horvath S, Mah V, Lu AT, Woo JS, Choi OW, Jasinska AJ, Riancho JA, Tung S, Coles NS, Braun J, et al. The cerebellum ages slowly according to the epigenetic clock. Aging (Albany NY). 2015;7(5):294-306.

23. Lowe D, Horvath S, Raj K. Epigenetic clock analyses of cellular senescence and ageing. Oncotarget. 2016;7(8):8524-31.

24. Raina A, Zhao X, Grove ML, Bressler J, Gottesman RF, Guan W, Pankow JS, Boerwinkle E, Mosley TH, Fornage M. Cerebral white matter hyperintensities on MRI and acceleration of epigenetic aging: the atherosclerosis risk in communities study. Clin Epigenetics. 2017;9:21,016-0302-6 eCollection 2017.

25. Hodgson K, Carless MA, Kulkarni H, Curran JE, Sprooten E, Knowles EE, Mathias S, Goring HHH, Yao N, Olvera RL, et al. Epigenetic age acceleration assessed with human White-matter images. J Neurosci. 2017;37(18):4735-43.

26. Singh MV, Chapleau MW, Harwani SC, Abboud FM. The immune system and hypertension. Immunol Res. 2014;59(1-3):243-53.

27. Daniels PR, Kardia SL, Hanis CL, Brown CA, Hutchinson R, Boerwinkle E, Turner ST. Genetic epidemiology network of Arteriopathy study. Familial aggregation of hypertension treatment and control in the genetic epidemiology network of Arteriopathy (GENOA) study. Am J Med. 2004; 116(10):676-81.

28. Aryee MJ, Jaffe AE, Corrada-Bravo H, Ladd-Acosta C, Feinberg AP, Hansen KD, Irizarry RA. Minfi: a flexible and comprehensive Bioconductor package for the analysis of Infinium DNA methylation microarrays. Bioinformatics. 2014;30(10):1363-9.

29. Fortin J, Triche TJ Jr, Hansen KD. Preprocessing, normalization and integration of the Illumina HumanMethylationEPIC array with minfi. Bioinformatics. 2016;33(4):558-60.

30. Lehne B, Drong AW, Loh M, Zhang W, Scott WR, Tan S, Afzal U, Scott J, Jarvelin M, Elliott P. A coherent approach for analysis of the Illumina HumanMethylation450 BeadChip improves data quality and performance in epigenome-wide association studies. Genome Biol. 2015;16(1):37.

31. Xu Z, Niu L, Li L, Taylor JA. ENmix: a novel background correction method for Illumina HumanMethylation450 BeadChip. Nucleic Acids Res. 2016;44(3):e20.

32. Houseman EA, Accomando WP, Koestler DC, Christensen BC, Marsit CJ, Nelson $\mathrm{HH}$, Wiencke JK, Kelsey KT. DNA methylation arrays as surrogate measures of cell mixture distribution. BMC Bioinformatics. 2012;13:86 2105-13-86.

33. Chen BH, Marioni RE, Colicino E, Peters MJ, Ward-Caviness CK, Tsai PC, Roetker NS, Just AC, Demerath EW, Guan W, et al. DNA methylation-based measures of biological age: meta-analysis predicting time to death. Aging (Albany NY). 2016;8(9):1844-65.

34. Lu AT, Xue L, Salfati EL, Chen BH, Ferrucci L, Levy D, Joehanes R, Murabito $J M$, Kiel DP, Tsai PC, et al. GWAS of epigenetic aging rates in blood reveals a critical role for TERT. Nat Commun. 2018;9(1):387,017-02697-5.

35. McEwen LM, Jones MJ, Lin DTS, Edgar RD, Husquin LT, Maclsaac JL, Ramadori KE, Morin AM, Rider CF, Carlsten C, et al. Systematic evaluation of DNA methylation age estimation with common preprocessing methods and the Infinium MethylationEPIC BeadChip array. Clin Epigenetics. 2008;10(1):123.

36. Levey AS, Stevens LA, Schmid CH, Zhang YL, Castro AF 3rd, Feldman HI, Kusek JW, Eggers $P$, Van Lente $F$, Greene $T$, et al. A new equation to estimate glomerular filtration rate. Ann Intern Med. 2009;150(9):604-12.

37. Jensen JS, Clausen P, Borch-Johnsen K, Jensen G, Feldt-Rasmussen B. Detecting microalbuminuria by urinary albumin/creatinine concentration ratio. Nephrol Dial Transplant. 1997;12(Suppl 2):6-9.

38. Kardia SL, Greene MT, Boerwinkle E, Turner ST, Kullo IJ. Investigating the complex genetic architecture of ankle-brachial index, a measure of peripheral arterial disease, in non-Hispanic whites. BMC Med Genet. 2008;1:16.

39. Arnett DK, Meyers KJ, Devereux RB, Tiwari HK, Gu CC, Vaughan LK, Perry RT, Patki A, Claas SA, Sun YV, et al. Genetic variation in NCAM1 contributes to left ventricular wall thickness in hypertensive families. Circ Res. 2011;108(3):279-83.

40. Devereux RB, Roman MJ, Ganau A, de Simone G, Okin PM, Kligfield P. Cardiac and arterial hypertrophy and atherosclerosis in hypertension. Hypertension. 1994;23(6 Pt 1):802-9.

41. Sahn DJ, DeMaria A, Kisslo J, Weyman A. Recommendations regarding quantitation in M-mode echocardiography: results of a survey of echocardiographic measurements. Circulation. 1978;58(6):1072-83.

42. Schiller NB, Shah PM, Crawford M, DeMaria A, Devereux R, Feigenbaum H, Gutgesell H, Reichek N, Sahn D, Schnittger I. Recommendations for quantitation of the left ventricle by two-dimensional echocardiography. American Society of Echocardiography Committee on standards, subcommittee on quantitation of two-dimensional echocardiograms. J Am Soc Echocardiogr. 1989;2(5):358-67.

43. Devereux RB, Lutas EM, Casale PN, Kligfield P, Eisenberg RR, Hammond IW, Miller $\mathrm{DH}$, Reis $\mathrm{G}$, Alderman MH, Laragh $\mathrm{JH}$. Standardization of M-mode echocardiographic left ventricular anatomic measurements. J Am Coll Cardiol. 1984;4(6):1222-30

44. Jack CR Jr, Twomey CK, Zinsmeister AR, Sharbrough FW, Petersen RC, Cascino GD. Anterior temporal lobes and hippocampal formations: normative volumetric measurements from MR images in young adults. Radiology. 1989;172(2):549-54.

45. Jack CR Jr, O'Brien PC, Rettman DW, Shiung MM, Xu Y, Muthupillai R, Manduca A, Avula R, Erickson BJ. FLAIR histogram segmentation for measurement of leukoaraiosis volume. J Magn Reson Imaging. 2001;14(6):668-76.

46. Smith JA, Turner ST, Sun W, Fornage M, Kelly RJ, Mosley TH, Jack CR, Kullo IJ, Kardia SL. Complexity in the genetic architecture of leukoaraiosis in hypertensive sibships from the GENOA Study. BMC Med Genet. 2009;2:16 8794-2-16.

47. Chillo P, Lwakatare J, Lutale J, Gerdts E. Increased relative wall thickness is a marker of subclinical cardiac target-organ damage in African diabetic patients. Cardiovasc J Afr. 2012;23(8):435-41.

48. Almasy L, Blangero J. Multipoint quantitative-trait linkage analysis in general pedigrees. Am J Hum Genet. 1998;62(5):1198-211.

49. Quach A, Levine ME, Tanaka T, Lu AT, Chen BH, Ferrucci L, Ritz B, Bandinelli S, Neuhouser ML, Beasley JM, et al. Epigenetic clock analysis of diet, exercise, education, and lifestyle factors. Aging (Albany NY). 2017;9(2):419-46.

50. Perna L, Zhang Y, Mons U, Holleczek B, Saum KU, Brenner H. Epigenetic age acceleration predicts cancer, cardiovascular, and all-cause mortality in a German case cohort. Clin Epigenetics. 2016:8:64,016-0228-z eCollection 2016

51. Regele F, Jelencsics K, Shiffman D, Pare G, McQueen MJ, Mann JF, Oberbauer R. Genome-wide studies to identify risk factors for kidney disease with a focus on patients with diabetes. Nephrol Dial Transplant. 2015; 30(Suppl 4):iv26-34.

52. Adler Al, Stevens RJ, Manley SE, Bilous RW, Cull CA, Holman RR, UKPDS GROUP. Development and progression of nephropathy in type 2 diabetes: the United Kingdom prospective diabetes study (UKPDS 64). Kidney Int. 2003;63(1):225-32

53. Palmieri V, Bella JN, Arnett DK, Liu JE, Oberman A, Schuck MY, Kitzman DW, Hopkins PN, Morgan D, Rao DC, et al. Effect of type 2 diabetes mellitus on left ventricular geometry and systolic function in hypertensive subjects: hypertension genetic epidemiology network (HyperGEN) study. Circulation. 2001;103(1):102-7.

54. Grant CD, Jafari N, Hou L, Li Y, Stewart JD, Zhang G, Lamichhane A, Manson JE, Baccarelli AA, Whitsel EA, Conneely KN. A longitudinal study of DNA methylation as a potential mediator of age-related diabetes risk. Geroscience. 2017:39(5-6):475-89.

55. Khan TH, Farooqui FA, Niazi K. Critical review of the ankle brachial index Curr Cardiol Rev. 2008;4(2):101-6.

56. Aboyans V, Criqui MH, Abraham P, Allison MA, Creager MA, Diehm C, Fowkes FG, Hiatt WR, Jonsson B, Lacroix P, et al. Measurement and 
interpretation of the ankle-brachial index: a scientific statement from the American Heart Association. Circulation. 2012;126(24):2890-909.

57. Marioni RE, Shah S, McRae AF, Chen BH, Colicino E, Harris SE, Gibson J, Henders AK, Redmond P, Cox SR, et al. DNA methylation age of blood predicts all-cause mortality in later life. Genome Biol. 2015;16:25.

58. Horvath S, Erhart W, Brosch M, Ammerpohl O, von Schönfels W, Ahrens M, Heits N, Bell JT, Tsai PC, Spector TD, et al. Obesity accelerates epigenetic aging of human liver. Proc Natl Acad Sci U S A. 2014;111(43):15538-43.

59. Gao X, Zhang Y, Breitling LP, Brenner H. Relationship of tobacco smoking and smoking-related DNA methylation with epigenetic age acceleration. Oncotarget. 2016;7(30):46878-89.

60. Beach SR, Dogan MV, Lei MK, Cutrona CE, Gerrard M, Gibbons FX, Simons RL, Brody GH, Philibert RA. Methylomic aging as a window onto the influence of lifestyle: tobacco and alcohol use Alter the rate of biological aging. J Am Geriatr Soc. 2015;63(12):2519-25.

61. Levine ME, Lu AT, Quach A, Chen BH, Assimes TL, Bandinelli S, Hou L, Baccarelli AA, Stewart JD, Li Y. An epigenetic biomarker of aging for lifespan and healthspan. Aging (Albany NY). 2018;10(4):573-91.

62. Liu Z, Chen BH, Assimes TL, Ferrucci L, Horvath S, Levine ME. The role of epigenetic aging in education and racial/ethnic mortality disparities among older U.S. Women. Psychoneuroendocrinology. 2019;104:18-24.

63. Sillanpaa E, Ollikainan M, Kaprio J, Wang X, Leskinen T, Kujala UM, Tormakangas T. Lesure-time physical activity and DNA methylation age-a twin study. Clin Epigenetics. 2019;11(1):12.

64. Maisel AS. Cardiovascular and renal surrogate markers in the clinical management of hypertension. Cardiovasc Drugs Ther. 2009;23(4):317-26.

\section{Publisher's Note}

Springer Nature remains neutral with regard to jurisdictional claims in published maps and institutional affiliations.

Ready to submit your research? Choose BMC and benefit from:

- fast, convenient online submission

- thorough peer review by experienced researchers in your field

- rapid publication on acceptance

- support for research data, including large and complex data types

- gold Open Access which fosters wider collaboration and increased citations

- maximum visibility for your research: over $100 \mathrm{M}$ website views per year

At BMC, research is always in progress.

Learn more biomedcentral.com/submissions 